\title{
Repeated Glucose Stimulation Reveals Distinct and Lasting Secretion Patterns of Individual Rat Pancreatic B Cells
}

\author{
Emanuele Giordano, Domenico Bosco, Vincenzo Cirulli," and Paolo Meda \\ Department of Morphology and *Laboratoires de Recherche "Louis Jeantet," University of Geneva \\ Medical School, CH-1211 Geneva 4, Switzerland
}

\begin{abstract}
To determine whether pancreatic B cells show a constant secretion pattern during repeated stimulations, we have used a sequential hemolytic plaque assay to monitor their individual insulin release during several successive 30 -min incubations in the presence of $16.7 \mathrm{mM}$ glucose. We have found that the total $B$ cell secretion did not vary significantly in these successive glucose stimulations and that, under these conditions, the majority of $\mathbf{B}$ cells that were stimulated to release insulin during the first incubation also secreted during the second, third, and, when this was tested, during the fourth incubation. Similarly, most of the $B$ cells that did not release detectable amounts of insulin during the first incubation did not secrete also during the two (or three) subsequent secretion tests. Together, the two groups of B cells that showed a constant secretory pattern, represented $\sim 75 \%$ of the entire $B$ cell population. The remaining $25 \%$ of $B$ cells shifted from a secreting to a non-secreting state, or vice versa, from one incubation to another. These observations were made under three different time frames in which we tested single $B$ cells as well as $B$ cell clusters at rather different intervals. These findings support the existence of distinct B cell subpopulations differing lastingly in their ability to secrete insulin in response to glucose. (J. Clin. Invest. 1991. 87:2178-2185.) Key words: glucose $\bullet$ heterogeneity $\bullet$ hemolytic plaque assay $\bullet$ insulin secretion $\bullet$ rat pancreatic B cells
\end{abstract}

\section{Introduction}

Within the islets of Langerhans, individual B cells differ markedly, in terms of organelle content $(1,2)$, extent of intercellular coupling (3), threshold for both glucose-induced electrical activity (4), and release of insulin-containing granules (5). Marked differences have also been observed in vitro between isolated $B$ cells in so far as the biosynthesis of new proteins (6), electrical activity $(7,8)$, and glucose-activated metabolic redox response (9) are concerned. Furthermore, using a hemolytic plaque assay for insulin, a wide heterogeneity in the secretory response of B cells to glucose has been reported (10-12). Thus, in preparations of islet cells stimulated by glucose, all intermediates were seen between $B$ cells that secreted huge amounts of insulin

Address reprint requests to Dr. Giordano, Department of Morphology, University of Geneva, C.M.U., 1, rue Michel Servet, CH-1211 Geneva 4, Switzerland.

Received for publication 25 July 1990 and in revised form 18 December 1990

J. Clin. Invest.

(c) The American Society for Clinical Investigation, Inc.

$0021-9738 / 91 / 06 / 2178 / 08 \quad \$ 2.00$

Volume 87, June 1991, 2178-2185 and B cells that did not secrete detectable amounts of the hormone, if they secreted at all (10-12).

The causes of this in vitro heterogeneity, under conditions in which all B cells are exposed simultaneously to the same environment (10-12), are unknown. Among the possibilities that should be envisaged, the most obvious are that either all B cells have the same ability to respond to glucose but do not secrete synchronously, or that there are distinct B cell subpopulations differing lastingly in their ability to release insulin after glucose stimulation. If the first hypothesis was true, the repeated exposure of a same B cell population to a stimulatory concentration of glucose should reveal the changing response of individual B cells that, with time, would fluctuate between a secreting and a non-secreting state. By contrast, if the second hypothesis was correct, the same test should reveal a more constant activity of B cells, most of which would be expected either to secrete repeatedly upon successive stimulations or to be repeatedly insensitive to the secretagogue. In order to address these possibilities, we have now developed a hemolytic plaque assay that permits to study the insulin secretion of individual B cells, during successive stimulations. Using this system, we have found that individual B cells show distinct secretory patterns and that, in a large majority, they reproduce a given secretory pattern during successive glucose stimulations.

\section{Methods}

Preparation of islet cells. Islets of Langerhans were isolated by collagenase digestion from the splenic portion of the pancreas of male Sprague-Dawley (SIVZ) rats weighing $250-350 \mathrm{~g}(5)$. The isolated islets were incubated for $15 \mathrm{~min}$ at room temperature in $1 \mathrm{ml}$ of a KrebsRinger-bicarbonate medium prepared without adding $\mathrm{Ca}^{2+}$ and supplemented with $11.2 \mathrm{mM}$ glucose, $0.5 \%$ bovine serum albumin (BSA), and 3 mM EGTA (10). At the end of this incubation, the islets were aspirated three times into a $2-\mathrm{ml}$ syringe through a 18 -gauge needle. This procedure was repeated using a 22-gauge needle and, finally, a 25-gauge needle. The resulting suspension, consisting of single cells and clumps of various size, was transferred into a rotating spinner flask and exposed for $3 \mathrm{~min}$ at room temperature to $1 \mathrm{mg} / \mathrm{ml}$ trypsin (1:250; Difco Laboratories, Inc., Detroit, MI) in the same Krebs-Ringer-bicarbonate $\mathrm{Ca}^{2+}$-free medium. The cells were then diluted with sterile RPMI 1640 medium (Gibco Laboratories, Grand Island, NY) supplemented with $10 \%$ fetal calf serum (FCS), $110 \mathrm{U} / \mathrm{ml}$ penicillin, and $110 \mu \mathrm{g} / \mathrm{ml} \mathrm{strep-}$ tomycin (RPMI medium), centrifuged for $10 \mathrm{~min}$ at $130 \mathrm{~g}$, and resuspended in RPMI medium. This procedure was repeated twice more before aliquots of $2 \times 10^{5}$ cells, suspended in $4 \mathrm{ml}$ of RPMI, were plated per 60-mm Petri dish to which cells do not adhere (no. 1007; Falcon Plastics, Oxnard, CA). Cells were cultured $12-18 \mathrm{~h}$ at $37^{\circ} \mathrm{C}$ in a humidified incubator gassed with air and $\mathrm{CO}_{2}$ to maintain medium pH at 7.4.

Sequential hemolytic plaque assay for insulin. The protocol we previously developed to quantitate insulin secretion using a reverse hemolytic plaque assay (10), was modified to permit the sequential study of very same B cells. Briefly, 50- $\mu$ l glass chambers coated with 0.1 $\mathrm{mg} / \mathrm{ml}$ of poly-L-lysine (mol wt 150,000-300,000; Sigma Chemical Co., St. Louis, MO) were prepared using a photoetched grid coverslip 
(no. 1916-92525; Bellco Glass, Inc., Vineland, NJ). Overnight cultured islet cells were diluted to a density of $5 \times 10^{4}$ cells $/ \mathrm{ml}$ in RPMI medium and $50-\mu \mathrm{l}$ aliquots of this preparation were injected per chamber. After a $45-$ min incubation at $37^{\circ} \mathrm{C}$ in an air $/ \mathrm{CO}_{2}$ incubator, during which cells adhered to the polylysine-coated glass, the chambers were thoroughly rinsed with RPMI medium and then filled with a modified Krebs-Ringer-bicarbonate medium supplemented with $4 \%$ protein Acoated sheep red blood cells (SRBC), $0.1 \%$ BSA, $0.1 \%$ ascorbic acid $16.7 \mathrm{mM}$ glucose, a specific and heat-inactivated $\left(45 \mathrm{~min}\right.$ at $\left.56^{\circ} \mathrm{C}\right)$ anti-insulin serum (13) diluted 1:50, and guinea pig complement diluted 1:50 (Behring Institut, Marburg, Federal Republic of Germany). This medium is hereafter referred to as test medium. After a 30-min incubation at $37^{\circ} \mathrm{C}$, the glass chambers were transferred to the stage of an inverted microscope (IM 35; Zeiss, Oberkochen, Federal Republic of Germany) and photographed at a magnification of 100 for the subsequent localization of individual cells and measurement of the hemolytic plaques they may have induced. $30 \mathrm{~min}$ later, once all photographs had been taken, the chambers were rinsed with RPMI medium to wash out completely the SRBC carpet (the position of islet cells, which were firmly attached to the poly-L-lysine-coated slides, did not change during this rinsing) and then refilled with test medium. After a second 30 -min incubation at $37^{\circ} \mathrm{C}$, the chambers were photographed again and the entire washing-refilling-incubating-photographing procedure repeated a third time. At the end of the third 30-min incubation, the chambers were filled with $0.02 \%$ Trypan blue in phosphatebuffered saline (PBS) and incubated for $10 \mathrm{~min}$ at room temperature. After rinsing with PBS, they were finally filled with Bouin's fixative.

A second series of experiments was performed according to the same protocol, except that the interval between each of the three successive 30-min incubations was set at $3 \mathrm{~h}$. In this case, once the assay chambers had been photographed, they were filled with RPMI medium and incubated at $37^{\circ} \mathrm{C}$ into a humidified incubator gassed with air and $\mathrm{CO}_{2}$ until the beginning of the next secretion test.

A third series of experiments was designed to test B cell secretion during another time frame by adding of a fourth incubation test after an interval which was not a multiple or a fraction of the intervals tested before. To this end, insulin release was assessed during four successive 30 -min incubations at intervals of 30,180 , and $75 \mathrm{~min}$, respectively. The rest of the protocol was otherwise identical to that of the two first experimental series.

Immunoidentification of $B$ cells. To identify the insulin-containing B cells, the Bouin's fixed chambers were rinsed in PBS, passed through a series of graded ethanols, and incubated for $2 \mathrm{~h}$ at room temperature with the same guinea pig anti-insulin serum which was used in the plaque assay test, diluted now 1:200 in PBS. Upon rinsing, the chambers were filled for $1 \mathrm{~h}$, at room temperature, with a fluoresceinlabeled rabbit serum against guinea pig IgG (Institut Pasteur, Paris, France) diluted 1:200 in PBS, rinsed in PBS, filled with a $0.02 \%$ p-phenylenediamine in a PBS/glycerine solution (1:2, vol/vol), and eventually sealed.

Quantitative analysis. Two to three experiments were performed according to each of the protocols to evaluate $(a)$ the percentage of single B cells and B cell clusters forming hemolytic plaques, $(b)$ the individual areas of these plaques, and $(c)$ the secretion pattern of individual B cells and clusters. To determine the proportion of cells surrounded by hemolytic plaques, about 200 single B cells and clusters were scored per experiment on the photographs taken at the end of each incubation. Counts were restricted to Trypan blue-excluding single B cells identified by a positive immunolabeling for insulin at the end of the last incubation. The same criteria were applied for scoring clusters, i.e., little aggregates containing no more than five $B$ cells and no other islet cell types. To evaluate the area of hemolytic plaques, photographs of these plaques were projected on a graphic tablet (model 4953, Tektronix, Inc., Beaverton, OR) connected to a IBM PC-XT 5150 programmed for automatic surface measurement. Data were computed separately for single B cells and B cell clusters. Total plaque development of single cells and clusters (14), was calculated by multiplying the values of the percentage of secreting cells and of the average hemolytic plaque area. Comparison of the photographs taken at the end of each of the three successive test incubations allowed us to identify repeatedly a series of very same cells and clusters, because of their constant position with respect to the grid photoetched on the coverslip. In this way, individual cells could be scored repeatedly for the presence or absence of a hemolytic plaque.

Percent data, average areas of hemolytic plaques and total plaque development values were expressed as mean \pm SEM and were compared using a Student's unpaired $t$ test.

\section{Results}

Fig. 1 shows part of a plaque assay chamber at the end of a 30 -min incubation in the presence of $16.7 \mathrm{mM}$ glucose. The individual position of single cells and clusters can be determined precisely, with respect to the letters and grid bars photoetched on the coverslip that formed the top of the assay chamber. As shown, the B cells that secreted insulin have become surrounded by a clear circular plaque of hemolysis, whereas other B cells that did not secrete detectable levels of insulin are still closely surrounded by a regular and dense carpet of intact red blood cells. Comparison of photographs taken at the end of each of the successive test incubations showed that islet cells were firmly attached to the floor of the chamber and did not modify their position during the assay, as judged by their constant relationships with the numbers and letters of the chambers' top (Fig. 2). These chambers were therefore used to assess repeatedly the secretion of a number of very same B cells during successive glucose stimulations.

To this end, we evaluated the proportion of single B cells and B cell clusters forming hemolytic plaques as well as the individual areas of these plaques, two parameters that reflect the number of secreting B cells and the amount of hormone they released, respectively $(10,15)$. As shown in Table $I$, the values evaluated for single $B$ cells did not change significantly between the first, second, and third incubation. The same was true for B cell clusters which gave values constantly higher $(P$ $<0.03$ ) than those evaluated for single B cells (Table I). The total insulin output of single cells and clusters was estimated by multiplying the values of the percentage of secreting cells and of the average hemolytic plaque area. As shown in Fig. 3, the resulting total plaque development of single B cells did not change significantly between the three successive secretion tests. The same was true for B cell clusters which also secreted about three times more $(P<0.03)$ than single $B$ cells throughout the experiment (Fig. 3). This increase is consistent with the average size of clusters which, in these experiments, comprised $2.8 \pm 0.1(n=8)$ B cells. The size distribution of clusters was similar from one experiment to another. Out of the 1,318 clusters that were scored, $52.5 \pm 3.7 \%$ comprised two B cells, $26.8 \pm 1.9 \%$ three B cells, $11.6 \pm 1.9 \%$ four B cells, and $8.9 \pm 2.2 \%$ five B cells.

When the secretory pattern of very same cells was analyzed repeatedly, we found that whereas some B cells secreted throughout the three incubations of each experiment, others never secreted detectable amounts of insulin ${ }^{1}$ and others, even-

1. To assess whether these $B$ cells were also insensitive to other secretagogues, preliminary experiments were performed by adding glucagon during the third incubation. Under these conditions, we observed that at least part of the B cells that did not secrete to a detectable level during the first two incubations in the presence of $16.7 \mathrm{mM}$ glucose could nevertheless release sizable amounts of insulin when subsequently stimulated by glucagon (E. Giordano, unpublished observation). 


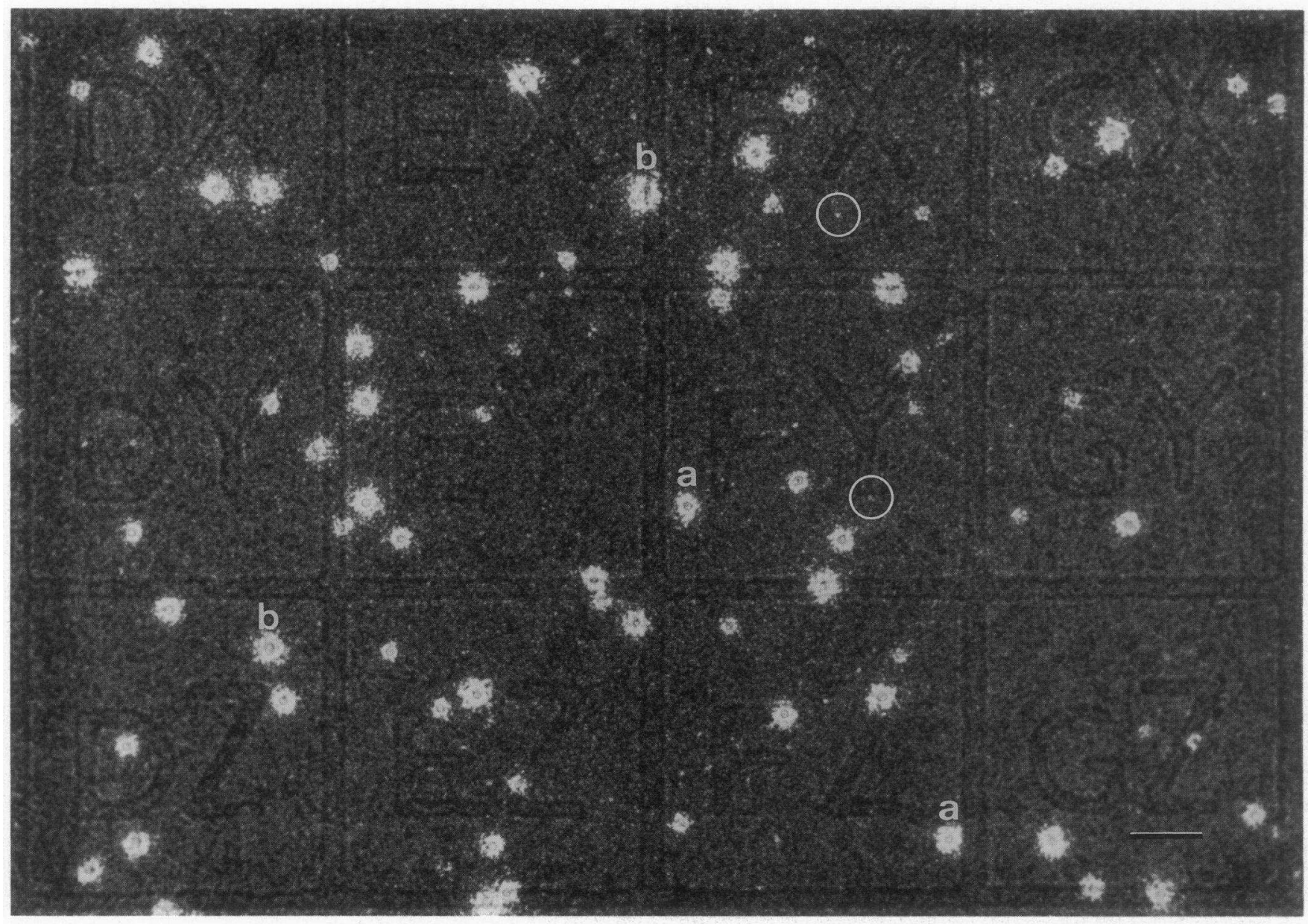

Figure 1. Low-magnification view of a plaque assay chamber after a 30-min stimulation by $16.7 \mathrm{mM}$ glucose. The position of individual B cells (a couple of which are labeled $a$ ) and B cell clusters (a couple of which are labeled $b$ ) can be determined precisely with respect to the letters and grid bars photoetched on the coverslip that forms the top of the chamber. Cells and clusters that have secreted insulin are surrounded by a hemolytic plaque which appears as a whitish round area within the dark grey carpet of intact red blood cells. Other islet cells (a couple of which are circled) are not surrounded by a hemolytic plaque, an indication that they did not secrete detectable amounts of insulin, if they secreted at all. Scale bar, $100 \mu \mathrm{m}$.

tually, secreted in only one or two of the three test incubations (Fig. 2). Scores showed that the majority of single cells and clusters retained throughout the experiment the secretory pattern they showed during the first incubation. Thus, $65.7 \pm 4.9 \%$ $(n=3)$ of the single B cells that secreted during the first incubation were also stimulated by glucose during the two successive secretion tests. In the same preparations, $91.8 \pm 0.2 \%$ of the B cells that did not elicit the formation of a hemolytic plaque during the first incubation also failed to secrete detectable amounts of insulin during the last two incubations of the experiment. Corresponding values for B cell clusters were $90.9 \pm 0.9 \%$ and $78.3 \pm 5.0 \%(n=3)$, respectively.

The $B$ cells that secreted constantly represented $36.6 \pm 4.4 \%$ of the 700 single and viable B cells that could be followed throughout the three successive glucose stimulations. The B cells that failed constantly to induce the formation of a hemolytic plaque represented $39.8 \pm 6.9 \%$ of this same population. Pooling these two groups revealed that $76.5 \pm 8.8 \%$ of single B cells showed a constant secretory pattern throughout the experiment, i.e., they were either repeatedly sensitive or repeatedly insensitive to glucose stimulation (Fig. 4). The proportion of these "constant" cells was significantly $(P<0.001)$ higher than that $(22.9 \pm 5.1 \%)$ of the remaining "fluctuating" B cells, that shifted from a secreting to a non-secreting pattern from one incubation to another (Fig. 4). Analogous observations were made with the $557 \mathrm{~B}$ cell clusters that could be identified at the end of the three successive test incubations of each experiment. $71.7 \pm 4.8 \%$ of these clusters secreted in each of these three incubations, whereas $16.2 \pm 2.4 \%$ never did so to a detectable level. Thus, the total proportion of clusters showing a constant secretion pattern $(87.9 \pm 1.1 \%)$ was significantly $(P<0.001)$ larger than that $(11.7 \pm 1.1 \%)$ of clusters showing a changing secretory response from one incubation to another (Fig. 4).

To assess whether these findings were dependent on the particular protocol we had chosen (three glucose stimulations 30 min apart) or could be reproduced within a different time frame, we repeated the experiments, leaving a 3-h interval between each of the three successive 30 -min glucose stimulations. The primary data evaluated under these new experimental conditions (Table II) showed that single B cells secreted in similar proportions and to a same extent in each of the three successive incubations. As a result, the total development of hemo- 

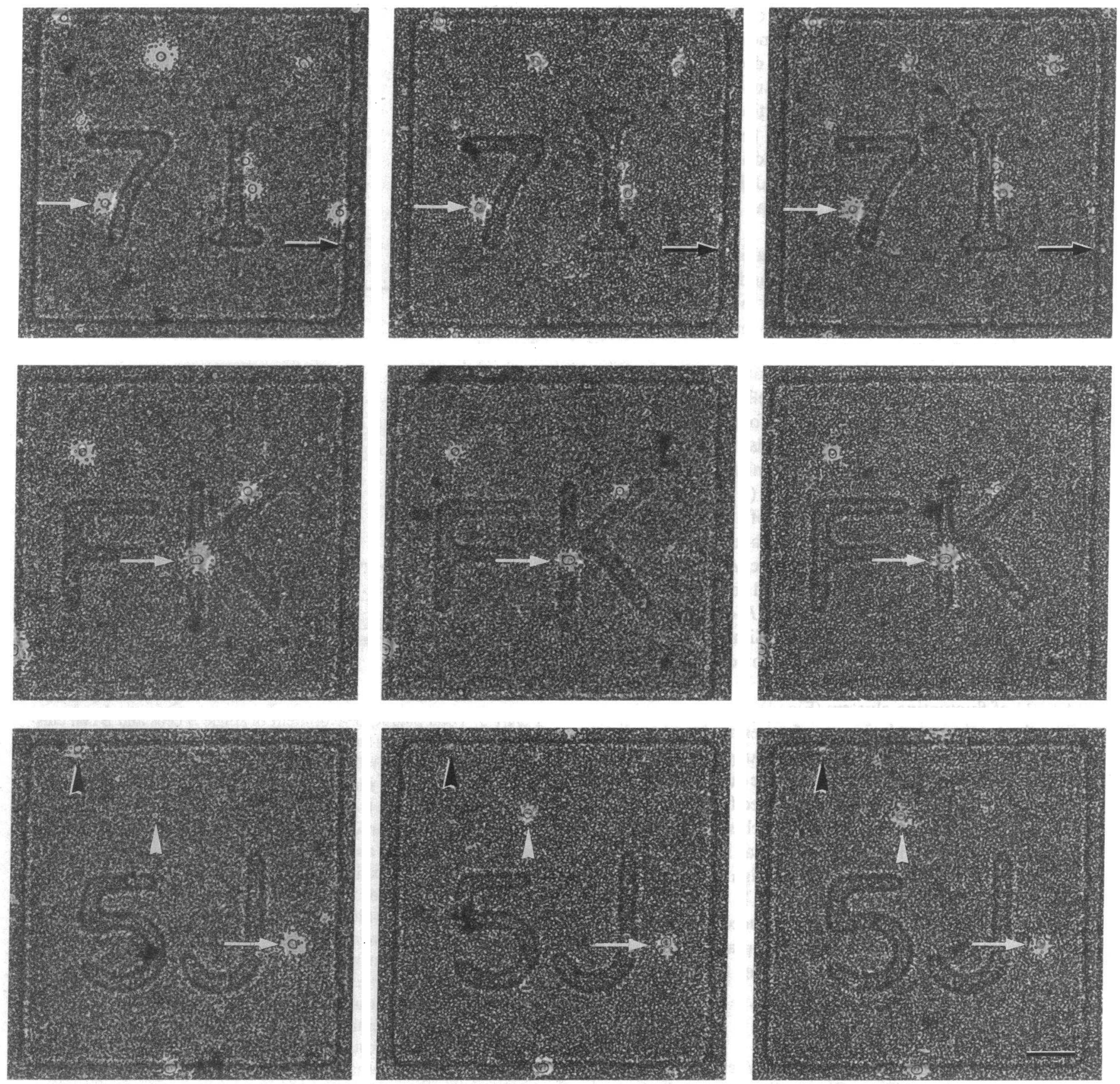

Figure 2. Three fields of a same plaque assay chamber are seen at the end of the first (left panel), second (middle panel), and third (right panel) 30 -min incubation in the presence of $16.7 \mathrm{mM}$ glucose. In field $7 I$ illustrated in the first row, a cell that secreted insulin in response to glucose all throughout the experiment (white arrow) coexists with a cell that did not form a hemolytic plaque during the three successive test incubations (black arrow). Both cells displayed the same secretory response ("on" and "off", respectively) throughout the experiment and were thus referred to as constant cells. Field $F K$ illustrated in the second row contains a small cluster (white arrow) of four B cells that also showed a constant secretion pattern. Field $5 J$ illustrated in the third row shows the coexistence within a same preparation of constant (white arrow) and fluctuating B cells (arrowheads). Among the latter cells, one (white arrowhead) did not form a hemolytic plaque during the first incubation but did secrete a sizable amount of insulin during the second and third incubations. The other cell (black arrowhead) secreted during the first but not during the second and third incubation. These two cells changed their secretory pattern during the three experimental tests and were therefore referred to as fluctuating B cells. Scale bar, $100 \mu \mathrm{m}$.

lytic plaques was not statistically modified throughout the experiment (Fig. 3). Analogous observations were made for B cell clusters (Table II), which also showed a total plaque development twice as high as that of single B cells (Fig. 3). As in the first series of experiments, the majority of $B$ cells showed a constant secretory pattern. Thus, $54.4 \pm 9.3 \%(n=3)$ of the single B cells and $67.6 \pm 10.7 \%$ of the $B$ cell clusters that elicited the formation of a hemolytic plaque during the first incubation continued to do so during the second and third incubations which took place 3 and $6 \mathrm{~h}$ later, respectively. Similarly, $76.6 \pm 4.3 \%$ of 
the single B cells and $75.7 \pm 3.4 \%(n=3)$ of the B cell clusters that did not elicit the formation of a hemolytic plaque at the beginning of the experiment also failed to do so during the two last exposures to glucose. Furthermore, analysis of individual secretory patterns showed that the proportion of constant cells $(68.4 \pm 1.2 \%)$ was larger $(P<0.001)$ than that $(31.4 \pm 1.3 \%)$ of fluctuating cells (Fig. 4). Similar conclusions were reached by comparing the proportion of constant and fluctuating clusters (Fig. 4), which represented $71.6 \pm 7.4 \%$ and $28.2 \pm 8.4 \%$ of the entire cluster population, respectively.

To assess whether B cells may be activated asynchronously, the insulin secretion of individual B cells and clusters was studied during four successive incubations, at intervals of 30, 180, and $75 \mathrm{~min}$, respectively. As in the two previous protocols, most of the 459 B cells scored showed a constant secretory behavior. The same was true for the 266 clusters counted. Thus, $78.8 \pm 7.4 \%(n=2)$ of the single B cells and $82.5 \pm 4.6 \%(n$ $=2$ ) of the B cell clusters that elicited the formation of a hemolytic plaque during the first incubation also did so during the second, third, and fourth incubations. Similarly, $70.4 \pm 1.3 \%$ ( $n$ $=2)$ of the single B cells and $62.6 \pm 0.5 \%(n=2)$ of the B cell clusters that did not elicit the formation of a hemolytic plaque at the beginning of the experiment also failed to do so during the three subsequent exposures to glucose. Analysis of the secretory pattern of individual cells showed that the proportion of constant cells $(70.2 \pm 2.7 \%)$ was larger $(P<0.001)$ than that $(29.8 \pm 2.7 \%)$ of fluctuating cells (Fig. 4). Similarly, the proportion of constant clusters, which represented $69.3 \pm 3 \%$ of the entire cluster population, was larger $(P<0.001)$ than that $(30.6 \pm 3 \%)$ of fluctuating clusters (Fig. 4 ).

The fluctuating population was further analyzed to distinguish the B cells that secreted insulin during two successive incubations from those that did not secrete during two consecutive incubations and from those that shifted from a secretory to a nonsecretory state, or vice versa, at each secretion test. The distribution of these cell groups was similar in the three protocols tested. Pooling of the data from the eight experiments performed showed that $6.1 \pm 1.6 \%$ of single B cells were able to induce a hemolytic plaque during two successive test incubations, $14.5 \pm 2.1 \%$ failed to induce such a plaque consecutively for two incubations, and $6.6 \pm 1.5 \%$ changed their secretory sta-

Table I. Primary Parameters Evaluated in the Hemolytic Plaque Assay for Insulin during Three Glucose Stimulations Performed 30 min Apart

\begin{tabular}{lccccc}
\hline & \multicolumn{2}{c}{ Single B cells } & & \multicolumn{2}{c}{ B cell clusters } \\
\cline { 2 - 3 } \cline { 5 - 6 } Incubation & Plaque forming & $\begin{array}{c}\text { Individual } \\
\text { plaque area }\end{array}$ & & Plaque forming & $\begin{array}{c}\text { Individual } \\
\text { plaque area }\end{array}$ \\
\hline & $\%$ & $\mu m^{2} \times 10^{3}$ & & $\%$ & $\mu m^{2} \times 10^{3}$ \\
First & $56.4 \pm 7.7$ & $1.0 \pm 0.1$ & & $78.7 \pm 5.3$ & $1.9 \pm 0.3^{*}$ \\
Second & $39.9 \pm 4.9$ & $0.9 \pm 0.1$ & & $74.0 \pm 5.6^{*}$ & $1.6 \pm 0.2^{*}$ \\
Third & $42.6 \pm 3.8$ & $1.0 \pm 0.1$ & & $77.3 \pm 4.0^{*}$ & $1.7 \pm 0.2^{*}$
\end{tabular}

Data were obtained at the end of three successive 30-min incubations in the presence of $16.7 \mathrm{mM}$ glucose performed $30 \mathrm{~min}$ apart. Values are expressed as mean \pm SEM of three separate experiments in which a total of 700 single B cells and 557 B cell clusters were scored.

$* P<0.03$ as compared to the corresponding value for single B cells.

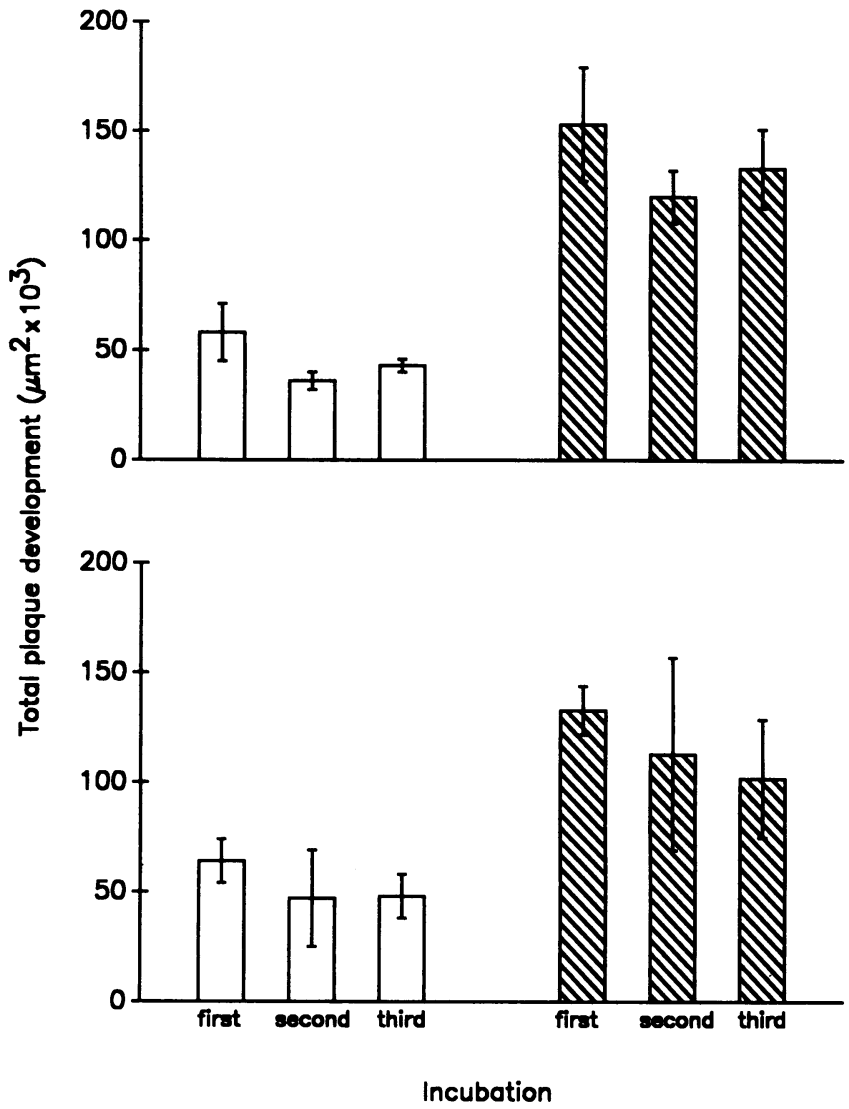

Figure 3. Total plaque development of single B cells and B cell clusters at the end of three successive 30 -min stimulations by $16.7 \mathrm{mM}$ glucose. (Upper panel) The values calculated for incubations performed $30 \mathrm{~min}$ apart show no significant change in the total plaque development of single B cells (open columns), suggesting a constant total insulin output throughout the experiment. B cell clusters (shaded columns) also showed a similar total plaque development during the three successive incubations. Throughout the experiment, their estimated total output was two to three times higher than that of single B cells. (Lower panel) Similar observations were made when the three glucose stimulations were performed $3 \mathrm{~h}$ apart. In this case again, total plaque development of both single cells (open columns) and clusters (shaded columns) was not significantly modified throughout the three successive incubations. As shown, the values of this parameter were virtually identical to those calculated for a shorter (30 $\mathrm{min}$ ) interval between incubations. In both upper and lower panels, total plaque development was calculated by multiplying the average area of hemolytic plaques by the proportion of plaque-forming B cells, and values for 100 single $B$ cells or B cell clusters were expressed as mean \pm SEM of three separate experiments.

tus at each incubation. Corresponding values for $B$ cell clusters were $6.3 \pm 1.3 \%, 10.4 \pm 2.1 \%$, and $5.3 \pm 1.3 \%$, respectively.

\section{Discussion}

We have developed a sequential hemolytic plaque assay to monitor repeatedly the insulin release of individual B cells, during successive exposures to stimulatory concentrations of glucose. We have first performed these test incubations at intervals of either $30 \mathrm{~min}$ or $3 \mathrm{~h}$, to test the functioning of the very same B cells over total periods of time of 2.5 and $7.5 \mathrm{~h}$, respec- 

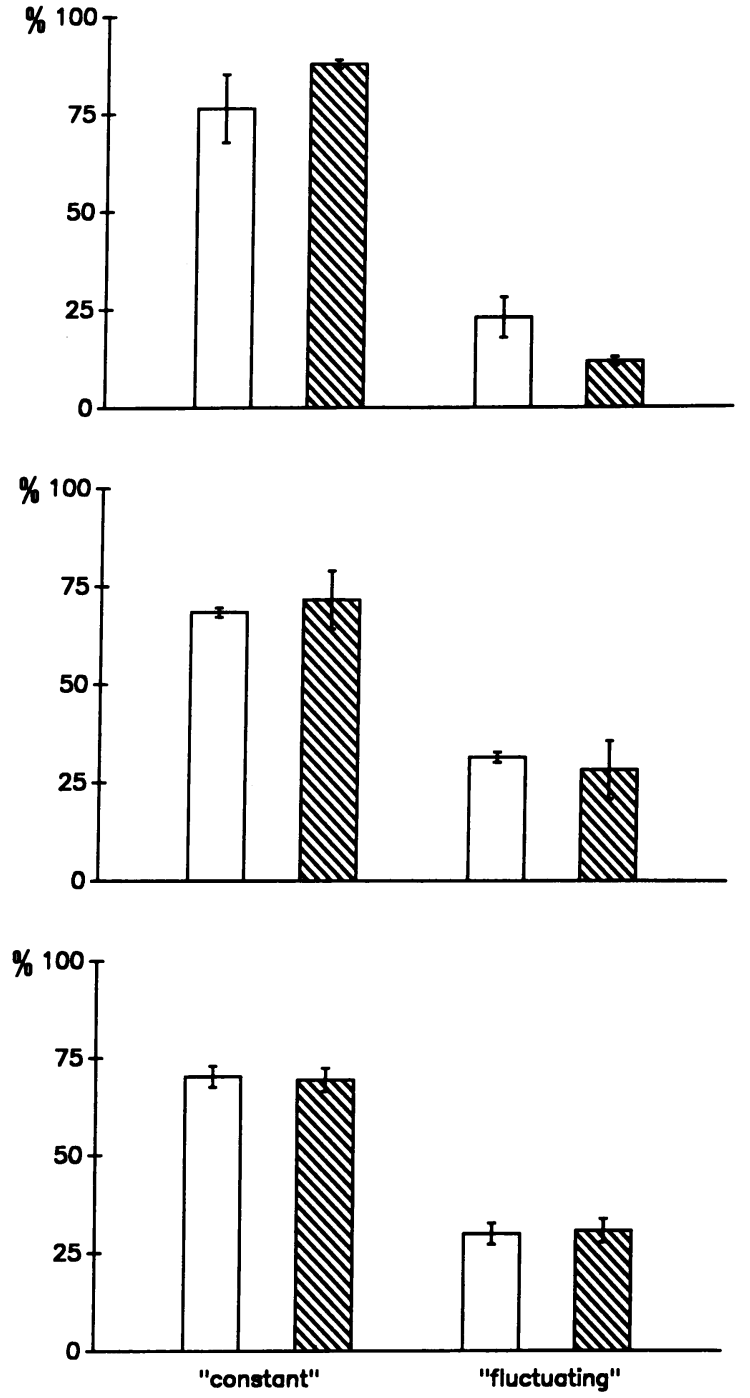

Figure 4. Proportion of single B cells and B cell clusters showing a constant and a fluctuating secretory pattern during several successive 30-min stimulations by $16.7 \mathrm{mM}$ glucose. (Upper panel) The percentage of single B cells (open columns) that either secreted or did not release detectable amounts of insulin during three successive incubations performed 30 min apart ("constant" cells), was about four times higher $(P<0.03)$ than that of B cells that secreted during only one or two of these incubations ("fluctuating" cells). The same observation was made for B cell clusters (shaded columns). (Middle panel) The proportions of constant and fluctuating cells were not modified when three glucose stimulations were performed $3 \mathrm{~h}$ apart. In this case again, a majority of both single cells (open columns) and clusters (shaded columns) were attributed to the constant group, which was significantly $(P<0.03)$ larger than the fluctuating group. (Lower panel) The proportion of constant and fluctuating B cells remained virtually the same also during four glucose stimulations performed at intervals of 30,180 , and $75 \mathrm{~min}$, respectively. With this time frame too, most of the single B cells (open columns) and B cell clusters (shaded columns) were attributed to the constant group, which was significantly $(P<0.03)$ larger than the fluctuating group. Values were expressed as mean \pm SEM of two (lower panel) or three (upper and middle panels) separate experiments. Cells were attributed to the constant group whenever they induced the development of a hemolytic plaque, or failed to do so, during all the successive incubations. By contrast, cells were attributed to the fluctuating group whenever they induced the formation of a hemolytic plaque in only some of the successive incubations.
Table II. Primary Parameters Evaluated in the Hemolytic Plaque Assay for Insulin during Three Glucose Stimulations Performed 3 h Apart

\begin{tabular}{lccccc}
\hline & \multicolumn{2}{c}{ Single B cells } & & \multicolumn{2}{c}{ B cell clusters } \\
\cline { 2 - 3 } \cline { 5 - 6 } Incubation & Plaque forming & $\begin{array}{c}\text { Individual } \\
\text { plaque area }\end{array}$ & & Plaque forming & $\begin{array}{c}\text { Individual } \\
\text { plaque area }\end{array}$ \\
\hline & $\%$ & $\mu m^{2} \times 10^{3}$ & $\%$ & $\mu m^{2} \times 10^{3}$ \\
First & $41.2 \pm 2.0$ & $1.6 \pm 0.3$ & & $61.6 \pm 4.6^{*}$ & $2.2 \pm 0.1$ \\
Second & $34.1 \pm 11.3$ & $1.3 \pm 0.2$ & & $53.1 \pm 12.2$ & $2.0 \pm 0.3$ \\
Third & $39.3 \pm 4.0$ & $1.2 \pm 0.1$ & & $54.2 \pm 8.5$ & $1.8 \pm 0.2$ \\
& & & & \\
\hline
\end{tabular}

Data were obtained at the end of three successive 30-min incubations in the presence of $16.7 \mathrm{mM}$ glucose performed $180 \mathrm{~min}$ apart. Values are expressed as mean \pm SEM of three separate experiments in which a total of 550 single B cells and 495 B cell clusters were scored.

* $P<0.01$ as compared to the corresponding value for single B cells.

tively. Within these two time frames, we have found that the total development of hemolytic plaques, which is a reflection of the entire insulin output of a B cell population $(14,16)$, was not significantly modified from one incubation to another. We have also observed that, as previously reported $(10-12,14,16)$, this unchanged total secretion was not contributed to a similar extent by each B cell but was actually the integrated result of individual secretory activities that differed markedly from one $B$ cell to another. Indeed, in all preparations, actively secreting $B$ cells coexisted with apparently non-secreting B cells, and all intermediates were seen between these two extremes. This apparent heterogeneity, ${ }^{2}$ under conditions in which all $B$ cells are exposed simultaneously to the same environment, could be due to the asynchronous activation of functionally alike $B$ cells, or to the existence of distinct $B$ cell subpopulations differing lastingly in their ability to release insulin in response to glucose.

To distinguish between these possibilities, we have monitored repeatedly individual B cells, in order to determine whether their secretory pattern changed in a random fashion from one incubation to another or, in contrast, remained the same throughout the experiment. We have found that most of the B cells that secreted actively during the first incubation remained secretory active also during the two successive stimulations. Similarly, the B cells that did not release detectable amounts of insulin, if they secreted at all, during the first stimulation also failed to secrete appreciably during the rest of the experiment. Together, the secreting and non-secreting B cells that showed a constant secretory pattern throughout the experiment, represented $\sim 75 \%$ of the entire B cell population, i.e., they were about three times more abundant than the B cells that turned from a secreting to a non-secreting state, or vice versa, from one incubation to another. The observation that "constant" B cells predominated "fluctuating" B cells in each

2. Virtually every $(99 \pm 0.9 \% ; n=2)$ B cell pair selected by cell sorting for glucose-activated metabolic redox response (9) forms a plaque of lysis under the conditions used in this study (V. Cirulli, unpublished data). This preliminary finding suggests that the absence of hemolytic plaques around some B cells most likely reflects their highly variable levels of insulin secretion rather than a methodological limit of the plaque assay. 
experiment and under two experimental protocols (stimulation every 30 or $180 \mathrm{~min}$ ) devised to test rather different time frames argues against a random fluctuation of B cells between an active and an inactive secretory state, as would be expected if all B cells were similarly sensitive to glucose but were releasing insulin asynchronously. To further dismiss such an asynchronous behavior, a protocol was designed to test B cells during a different time frame, including an interval which was not a multiple or a fraction of the intervals tested before. Again, in this protocol constant B cells predominated fluctuating B cells. Irrespective of the protocol chosen, the three subcategories of B cells that were distinguished in the fluctuating population were similarly represented from one experiment to another. These data suggest that a previous exposure to a stimulatory level of glucose is not a prerequisite for a subsequent sugar-induced stimulation of insulin release, at least for a sizable proportion of B cells. Although these data do not exclude the possibility that the threshold for glucose-induced insulin secretion may be modulated at the level of individual B cells, they provide direct evidence that this threshold is not markedly lowered in the majority of $\mathbf{B}$ cells during repeated exposures to the sugar.

Taken together, these data support the existence of B cell subpopulations differing markedly in their ability to release insulin and able to reproduce a given secretory pattern lastingly, and during repeated challenges by their major natural secretagogue. This tentative conclusion raises questions as to the factors that may modulate differentially the glucose sensitivity of individual B cells. Insulin-producing cells have already been shown to differ in terms of threshold for glucose-stimulated electrical activity $(4,7,8)$, protein biosynthesis $(6,17)$, metabolic redox state (9), and secretagogue-induced oscillations of intracellular $\mathrm{Ca}^{2+}$ concentrations (18). However, whether any of these apparently intrinsic biophysical and metabolic characteristics are related to, or may be even responsible for the differences we have observed in the secretory behavior of individual B cells, remains to be established. It is also uncertain why some B cells appear unable to release insulin, at least to any significant level, when properly and repeatedly stimulated by glucose. We have recently obtained evidence that these B cells are equipped with the same glucose transporter (D. Bosco, unpublished observation) and ionic membrane channels than secreting B cells, and share with them the same secretagogue-induced changes of major electrical conductances (19). These two observations suggest that at least the first steps of the mechanism that normally couples the stimulus by glucose to insulin secretion (20) are preserved in the B cells which cannot induce the formation of a hemolytic plaque. That the more distal steps of the secretory machinery of these cells can also be activated is suggested by our preliminary observation that at least some of these glucose-insensitive B cells can be induced to form an insulin-dependent hemolytic plaque when stimulated by glucagon. Therefore, these cells appear to have a normal secretory machinery which is not properly activated by glucose.

Contact-dependent cell interactions may play a role in this defect. Indeed, as in previous studies $(10,14,16)$, we have observed that the proportion of B cell clusters secreting insulin is significantly larger than that of single B cells. Actually, this proportion is larger than that which can be computed, assuming that B cells reaggregate at random and that contact has no influence on their individual secretory pattern. These observations suggest that at least some of the single B cells that are insensitive to glucose do recognize the sugar as a stimulus for insulin release after contacting other B cells. Whatever the contact-dependent mechanism responsible for such an important change $(10,14,16)$, it did not, however, permit to recruit all $B$ cells to secrete lastingly. Thus, at least in vitro, some functionally intact B cells appear repeatedly unable to respond to glucose, including after they have become part of a cluster.

It remains to be established whether these in vitro findings have any relevance for in vivo conditions. We have previously shown that, within individual islets of Langerhans, all B cells do not release insulin at the same rate during glucose infusion (5). Strikingly, the B cells located in the center of the islets release insulin-containing secretory granules much faster than the B cells that are located at the islet periphery and that began to degranulate only after the glucose infusion had been sustained for several hours (5). Whether these peripheral B cells correspond to the glucose-insensitive B cells detected in the plaque assay and, at any rate, what may be their role in the islet cell community are presently two completely opened questions.

In summary, we have shown that individual B cells differ markedly in their ability to release insulin when challenged by glucose, and that these functional differences are maintained steadily during successive stimulations spanning variable periods of time. As in other gland systems in which secretory cells have been shown to be functionally heterogeneous (21-25), it is possible that the existence of $B$ cell subpopulations with constant but distinct functional characteristics may be of importance to regulate precisely, in a graded and moment-to-moment way, the amount insulin needed to be secreted in order to match the ever-changing requirements of the organism. To test this hypothesis, an assessment of how the ratio of secreting to non-secreting B cells may be affected under other experimental conditions, such as after other time intervals and/or in the presence of various concentrations of different secretagogues is now required. The approach we report here now permits addressing these questions.

\section{Acknowledgments}

We thank L. Burkhardt, A. Charollais, S. De Mitri, J.-P. Gerber, L. Juliano, and P. Ruga for technical assistance.

This work was supported by grants 31-26625.89 and 3100-009394 from the Swiss National Science Foundation, 187384 from the Juvenile Diabetes Foundations International, and from the Sir Jules Thorn Charitable Overseas Trust Rep., Schaan.

\section{References}

1. Orci, L. 1974. A portrait of the pancreatic B-cell. Diabetologia. 10:163-187. 2. Meda, P., J.-F. Denef, A. Perrelet, and L. Orci. 1980. Nonrandom distribution of gap junction between pancreatic B-cells. Am. J. Physiol. 238:C114-C119.

3. Michaels, R. L., and J. D. Sheridan. 1981. Islets of Langerhans: dye coupling among immunocytochemically distinct cell types. Science (Wash. DC). 214:801-803.

4. Beigelman, P. M., B. Ribalet, and I. Atwater. 1977. Electrical activity of mouse pancreatic beta-cells. II. Effects of glucose and arginine. J. Physiol. (Paris). 73:201-217.

5. Stefan, Y., P. Meda, M. Neufeld, and L. Orci. 1987. Stimulation of insulin secretion reveals heterogeneity of pancreatic B cells in vivo. J. Clin. Invest. 80:175-183.

6. Schuit, F. C., P. A. In 'T Veld, and D. G. Pipeleers. 1988. Glucose stimulates proinsulin biosynthesis by a dose-dependent recruitment of pancreatic beta cells. Proc. Natl. Acad. Sci. USA. 85:3865-3869.

7. Meda, P., I. Atwater, A. Goncalves, A. Bangham, L. Orci, and E. Rojas. 1984. The topography of electrical synchrony among B-cells in the mouse islet of Langerhans. Q. J. Exp. Physiol. 69:719-735. 
8. Perez-Armendariz, E., I. Atwater, and E. Rojas. 1985. Glucose-induced oscillatory changes in extracellular ionized potassium concentration in mouse islets of Langerhans. Biophys. J. 48:741-749.

9. Pipeleers, D., and M. Van de Winkel. 1989. Cellular endogenous fluorescence: A basis for preparing subpopulations of functionally homogeneous cells. In Cell Structure and Function by Microspectrofluorometry. E. Kohen and J. G. Hirschberg, editors. Academic Press Inc. 391-404.

10. Salomon, D., and P. Meda. 1986. Heterogeneity and contact dependent regulation of hormone secretion by individual B cells. Exp. Cell Res. 162:507520 .

11. Hiriart, M., and R. Matteson. 1988. $\mathrm{Na}^{+}$channels and two types of $\mathrm{Ca}^{2+}$ channels in rat pancreatic B-cells identified with the reverse hemolytic plaque assay. J. Gen. Physiol. 91:617-639.

12. Lewis, C. E., A. Clark, S. J. H. Ashcroft, G. J. S. Cooper, and J. F. Morris. 1988. Calcitonine gene-related peptide and somatostatin inhibit insulin release from individual rat B cells. Mol. Cell. Endocrinol. 57:41-49.

13. Wright, P. H., D. R. Makalu, and I. J. Posey. 1968. Guinea pig anti-insulin serum. Diabetes. 17:513-516.

14. Bosco, D., L. Orci, and P. Meda. 1989. Homologous but not heterologous contact increases the insulin secretion of individual pancreatic B-cells. Exp. Cell Res. 184:72-80.

15. Allaerts, W., A. Wouters, D. Van der Massen, A. Peerson, and C. Denef. 1988. A diffusion-adsorption model for the computation of the amount of hormone around a secreting cell, detected by the reverse hemolytic plaque assay. $J$. Theor. Biol. 131:441-459.

16. Meda, P., D. Bosco, M. Chanson, E. Giordano, L. Vallar, C. Wollheim and L. Orci. 1990. Rapid and reversible secretion changes during uncoupling of rat insulin-producing cells. J. Clin. Invest. 86:759-768.
17. Meda, P., and D. Bosco. 1989. Actively synthesizing B-cells secrete preferentially after glucose stimulation. Diabetologia. 32:516a. (Abstr.)

18. Prentki, M., M. C. Glennon, A. P. Thomas, R. L. Morris, F. M. Matschinsky, and B. E. Corkey. 1988. Cell-specific patterns of oscillating free $\mathrm{Ca}^{2+}$ in carbamylcholine-stimulated insulinoma cells. J. Biol. Chem. 263:1 1044-11047.

19. Soria, B., M. Chanson, and P. Meda. 1989. Glucose responsive and unresponsive B-cells show similar electrical characteristics. Diabetologia. 32:543A (Abstr.)

20. Prentki, M., and F. M. Matschinsky. 1987. $\mathrm{Ca}^{2+}$, cAMP, and phospholipid-derived messengers in coupling mechanisms of insulin secretion. Physiol. Rev. 67:1185-1248.

21. Boockfor, F. R., J. P. Hoeffler, and L. S. Frawley. 1986. Analysis by plaque assay of GH and prolactin release from individual cells in cultures of male pituitaries. Neuroendocrinology. 42:64-70.

22. Keith, L. D., B. Tam, H. Ikeda, Z. Opsahl, and M. A. Greer. 1986. Dynamics of thyrotropin-releasing hormone-induced thyrotropin and prolactin secretion by acutely dispersed rat adohypophyseal cells. Neuroendocrinology. 43:445-452.

23. Lin, C.-T., W. Palmer, J.-Y. Wu, and L. Chan. 1986. Estrogen induction of very low density apolipoprotein II synthesis, a major avian liver yolk protein, involves the recruitment of hepatocytes. Endocrinology. 118:538-551.

24. Studer, H., H. J. Peter, and H. Gerber. 1989. Natural heterogeneity of thyroid cells: the basis for understanding thyroid function and nodular goiter growth. Endocr. Rev. 10:125-135.

25. Boockfor, F. R., L. K. Schwarz, and F. C. Derrick III. 1989. Sertoli cells in culture are heterogeneus with respect to transferrin release: analysis by reverse hemolytic plaque assay. Endocrinology. 125:1128-1133. 